\title{
Reformismus oder Sozialfaschismus?
}

\author{
Antwort auf Niels Kadritzkes Polemik in PROKLA Nr. 11/12
}

\section{Alexander von Plato}

\begin{abstract}
Der für meine Antwort zur Verfugung gestellte Raum ist klein und meine Vorbereitungszeit kurz - deshalb will ich sofort zum Kern der Auseinandersetzung kommen und nur am Rande auf unwissenschaftliche Fälschungen und die teilweise präffische Polemik (1) Kadritzkes eingehen.
\end{abstract}

1 Die gröbsten Fälschungen, die sich Niels Kadritzke bei der Darstellung meiner Arbeit leistet:

Ich hätte Trotzkis Position, ,auf unzulässige Weise gekürzt", insbesondere, ,fehit vor allem der Hinweis auf Trotzkis Hauptforderung für eine kommunistische Einheitsfrontpolitik, nämlich ein konkretes Programm mit der sozialdemokratischen Basis selbstverständlichen Forderungen anstelle eines bürokratischen Ultimatismus" (PROKLA 1/1974, S. 61, Fn.9). Ich kenne keine Arbeit über Trotzki, die dermaßen ausführlich Trotzkis Positionen referiert, was mir den Vorwurf seitens der KPD/ML-Genossen eingebracht hat, Propaganda für den Trotzkismus zu betreiben. Offensichtlich hat Niels meine Arbeit nicht vollständig gelesen, denn gerade auf die meiner Ansicht nach weit hinter die präzisen betrieblichen Einheitsfrontprogramme der KPD zurückfallenden Forderungen Trotzkis gehe ich ausführlich en, auch auf seine sonstigen Forderungen für eine Einheitsfront (RGO, Zusammengehen auf allen Ebenen usw.), vgl. folgende Seiten meiner Arbeit: S. 83 ff., S. 88 ff., S. 239 und S. 240.

Des weiteren behauptet Kadritzke, ich hätte die unterschiedlichen Begründungen für den Sozialfaschismus in den Theorien der KPD nicht gesehen: einmal hätte die KPD die SPD-Führung selbst als Träger einer sozialfaschistischen Diktatur über die Arbeiterklasse gesehen, dann als Wegbereiter des Faschismus oder als Teil einer faschistischen Diktatur. Wiederum muß Kadritzke sich den Vorwurf gefallen lassen, das nicht gründlich gelesen zu haben, was er kritisiert. Diese Unterschiede in der Einschätzung des Sozialfaschismus beschreibe ich sehr wohl, vgl. S. 206, 333. Ich meine aber, daß diese Unterschiede nicht einfach Änderungen in den Häuptern der KPD-Theoretiker waren, sondern reale Möglichkeiten angesichts der Politik der SPD-Führer.

Außerordentlich unwissenschaftlich ist es, wenn aus den zahlreichen Äußerungen der heutigen KPD zum "Programm zur nationalen und sozialen Befreiung des deutschen Volkes" gerade die eine herausgefischt wird, in der die Reihenfolge von ,national" und "sozial" vertauscht wurde. Und daraus wird dann auch noch eine Ideologie des ,KSV gemacht. Im übrigen gehe ich sehr ausführlich auf dieses Programm ein, im Gegensatz zu Kadritzkes Behauptung, vgl. S. $256 \mathrm{ff}$.

Gerade gefährlich finde ich es, wenn meine Behauptung, die ADGB-Führer kämen in der Frage der Abschaffung der Frauenarbeit der faschistischen Ideologie sehr nahe, mit den „reformistischen", „, naiven" Argumenten beantwortet wird: der ADGB habe sich von der Abschaffung der Frauenarbeit Arbeitsplätze wenigstens für Männer versprochen, mann wollte damit der Lohndrückerei begegnen, als ob damit nicht Löhne für eine Arbeiterfamilie gedrückt werden. Diese „Begründung" der ADGB-Führer war reine Demagogie und unterscheidet sich eben kaum von der faschistischen Ideologie.

Kadritzke behauptet, ich hätte die sozialdemokratischen Lohntheorien von Braunthal 
Der Kern, um den es geht, scheint mir folgender zu sein: Ist die Politik der sozialdemokratischen Führer seit $1914 \mathrm{im}$ Wesen eine Politik des Imperialismus, die große Teile der Arbeiterklasse mithilfe reformistischer Phrasen und in Phasen relativer Stabilität auch mit tatsächlichen Zugeständnissen an den kapitalistischen Staấ ketten soll - oder eine Politik des Reformismus, wie Kadritzke meint. Welche Politik betreibt eine sozialdemokratische Parteiführung, wenn das System, mit dem sie auch materiell verwachsen ist, in tiefe ökonomische und politische Krisen gerät und nur noch durch den brutalen Terror über die Arbeiterklasse seine Herrschaft aufrechterhalten kann? Konkret: wie verhielt sich die SPD-Führung zwischen 1928 bis 1933 während der umfassenden Krise und schließlich während des drohenden Faschismus? War der Terrorismus der SPD-Führer nur der widersprüchliche Ausrutscher von Reformisten oder die klare Antwort auf die Alternative: ,Ja“ zum immer terroristischer werdenden Staat oder ,Ja" zum Kampf um Reformen, auch mit Kommunisten, um demokratische Rechte und gegen die ökonomische Verelendung im bürgerlichen Staat? Niels Kadritzke bezeichnet die Politik der SPD-Führung in seinem Aufsatz als reformistisch. Daher resultiert auch

\section{Kadritzkes 1. These}

Die SPD-Führer waren nur insofern ,mitschuldig am Sieg des Faschismus“, als sie in Passivität, Hilflosigkeit und Kapitulantentum angesichts des Scheiterns ihrer Reformpolitik die Arbeiterklasse vom Kampf gegen den Faschismus abhielten.

Dagegen setze ich aus der unterschiedlichen Einschätzung und Untersuchung des Wesens der Politik der SPD-Führer die Feststellung: Die SPD- und ADGB-Führung beteiligte sich aktiv und terroristisch und zu Anfang der Krise in Regierungsverantwortung an der politischen und ökonomischen Verelendung der Arbeiterklasse, half mit bei der Aushöhlung des bürgerlich-demokratischen Staates und ebnete dem Faschismus den Weg - ja, zumindest die Gewerkschaftsfïhrung war zu einer offenen Arbeitsteilung mit dem regierenden Faschismus bereit. Denn: die SPDGewerkschaftsfiuhrung kettete sich an einen Klassenstaat, der sich in der Krise nur noch durch den nackten Terror gegenüber der revolutionären und schließlich auch gegenüber der reformistischen Erfolge der Arbeiterbewegung halten konnte. An einen Staat also, der immer mehr faschistische Elemente zu seiner Herrschaftssicherung aufnahm und der sich "legal und friedlich" in den faschistisch regierten bürgerlichen Staat , verwandelte“.

Da Kadritzke das Wesen der Politik der SPD-Führung als reformistisch begreift, legt er die Vermutung nahe, die SPD-Führung hätte während dieses Prozesses

und den Nöltings gleichgesetzt, ,obwohl diese genau gegenteilige Forderungen begründe-

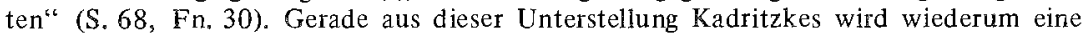
Ideologie der Verwischung von Widersprüchen meinerseits gemacht, was absurd ist angesichts der Tatsache, daß ich sehr genau auf die Unterschiede zwischen diesen sozialdemokratischen Lohntheorien eingehe. Vg1. S. 174 f. meiner Arbeit.

Die Stoßrichtung der Fälschungen und Unterstellungen Kadritzkes ist eindeutig und zeigt die unwissenschaftliche Manier, mit der hier politisch gearbeitet wird. 
der ,Faschisierung ${ }^{66}$ um der ,Erhaltung der Demokratie ${ }^{66}$ willen nur eine verheerende ,Kompromißpolitik ${ }^{66}$ betrieben.

Deshalb hängt alles an đer Frage: Beteiligte sich die SPD- und ADGB-Führung aktiv und terroristisch an der Aushöhlung der Weimarer Republik, nahm sie Elemente der faschistischen Ideologie und Praxis auf und war sie zu einer Arbeitsteilung mit dem regierenden Faschismus bereit oder kapitulierte sie nur passiv vor dem Faschismus? Diese Frage muß untersucht werden nicht nur anhand der Theorien der sozialdemokratischen Führung, sondern vorrangig anhand ihrer Praxis:

Von 1928 bis 1930 regierte eine Koalitionsregierung unter dem Sozialdemokraten Hermann Müller den Weimarer Staat, zeichnete also verantwortlich für die ungeheure Verelendung der Arbeiterklasse während dieser Zeit. Keine bürgerliche Regierung konnte in so kurzer Zeit ein solches arbeiterfeindliches Programm durchsetzen wie die Regierung Müller:

Sie belastete durch Erhöhung der direkten und indirekten Massensteuern die Werktätigen in knapp zwei Jahren mit 1.245 Milliarden Reichsmark;

gleichzeitig strich oder senkte sie die Kapitalistensteuern um 1.37 Milliarden Mark, nämlich die Gewerbe-, Grund-, Gebäude-, Einkommens-, Vermögensund Hauszinssteuern, sowie die Industriebelastungen und die Rentenbankschulden (1a);

- die Arbeitslosigkeit stieg von 9,7\% (1928) auf 22,7\%(1930) (2);

- die Arbeitsleistung eines Arbeiters pro Stunde stieg bei einem zugrundegelegten Index von 1913 = 100 von 119 (1928) auf 125 (1930) (3);

- im Oktober 1929 wurde durch Müllers Arbeitsminister Wissell (einem sozialdemokratischen ADGB-Führer) die Arbeitslosenunterstützung gekürzt, so daß 1930 über eine Million Arbeitslose nicht unterstützt wurden. Gleichzeitig wurde die Arbeitslosenversicherung um 3,5\% erhöht - eine weitere Mehrbe. lastung der Werktätigen (4);

- der Achtstundentag wurde faktisch abgeschafft; Arbeitszeiten von 14 und 15 Stunden wurden nicht selten bei den Aufsichtsämtern registriert (5);

- der Lohn sank von 1928 bis 1930 um 10.60 RM pro Woche unter das Existenzminimum (6);

- während die Winterbeihilfe bei den Stimmen der SPD gegen die KPD abgelehnt und die Kinderspeisung, fur die die SPD in den Wahlkampf gezogen war, um 5 Millionen RM gestrichen wurde, wurde der Wehretat gleichzeitig um 50 Millionen RM erhöht (6a).

la Inprekorr, Nr. 50, vom 13.6.1930, S. 1107. Vgl. auch: Th. Neubauer. Zu Hilferdings Steuerplänen, in: Die Internationale, Nr. 3/1929, oder ders.: Die zweite Aera Hilferding, ebd., Heft 20

2 Horst Geyer. Die Arbeiterbewegung und Gegenkräfte in Gesellschaft und Staat (1871-1933), Arbeismaterialien der IG CPK, Hannover, o. J., S. 59

3 J. Kuczynski. Geschichte der Lage der Arbeiterklasse, Bd. 5, Berlin/DDR 1966, S. 207

4 Inprekorr, 1930, Nr. 9, S. 214

5 Kuczynski, a. a. O., S. 201

6 Ebd., S. 222

6a Erika König: Vom Revisionismus zum „Demokratischen Sozialismus", Berlin/DDR 1964 , S. 166 
In Erwartung der kommenden Unruhen erklärte Reichskanzler Müller:

„Allen Bestrebungen, die politische Unruhen hervorrufen wollen (!), muß scharf entgegengetreten werden. Die Furcht vor Unruhen fördert die Kapitalflucht, aber die Regierung ist stark genug, solchen Bestrebungen Halt zu gebieten" (7).

Diese Marschroute zur politischen Unterdrückung der Arbeiterklasse und zur Durchsetzung der Abwälzung der Krise auf die Werktätigen hielt die Regierung Müller auch ein:

Was keiner Regierung zuvor gelungen war, schaffte die Regierung unter dem Sozialdemokraten Müller: das Verbot der Militärorganisation der KPD, des Roten Frontkämpferbundes, während gleichzeitig die Faschisten noch ,legal ${ }^{\circ}$ ihre Stoßtruppen aufbauen konnten. Das war eine wichtige Vorbedingung für den Sieg der Faschisten und ihrer Stoßtrupps, die auch unter ,illegalen“ Bedingungen mit dem Wohlwollen der Staatsbürokratie rechnen konnten.

- Das 2. Republikschutzgesetz - lange verschoben - wurde unter der Regierung Müller verabschiedet. Das war wiederum eine wichtige Vorbedingung für den Siegeszug der Faschisten, denn sie wurden von der späteren Rechtsprechung kaum betroffen; sehr wohl aber Kommunisten und Antifaschisten, später auch Sozialdemokraten.

- Schießbefehle wurden von den Reichs- und Provinzregierungen ausgegeben und häufig genug auch ausgefuihrt. Nicht nur bei dem berüchtigten Blut-Mai von 1929, wo während der von der SPD-Regierung verbotenen Mai-Demonstration 31 Arbeiter von der Zörgiebel-Polizei erschossen wurden. Auch in anderen Städten, in denen SPD-Polizeipräsidenten herrschten, wie in Kiel, Hamburg, Leipzig, in Worms, Chemnitz, im Ruhrgebiet und vielen anderen Städten, wurde auf verbotenen Hungermärschen arbeitsloser Arbeiter von der Schußwaffe Gebrauch gemacht, zig Arbeiter fanden in diesen Jahren den Tod auf der Straße. Es herrschte - wie der Komintern-Funktionär Manuilski feststellte - „ein Unternehmerterror, wie er selbst im zaristischen Rußland nicht bestanden" habe (8). Nach dem Blut-Mai von Berlin wurde das Demonstrationsverbot wieder aufgehoben, so daß dem Aufmarsch des ,Stahlhelm" nichts mehr im Wege stand.

- Ein weiteres Beispiel für den rigorosen Abbau normalster demokratischer Rechte durch die SPD-Regierung ist der Erlaß des SPD-Innenministers von Preußen, Grzesinski: danach sollten regulär gewählte kommunistische Bürgermeister nicht anerkannt werden (8a).

- Außerdem wurden ständig Kommunisten verfolgt, das KPD-Liebknecht-Haus mehrfach besetzt und auch vor der Abgeordneten-Immunität kommunistischer Abgeordneter nicht zurückgeschreckt.

Das alles ist zwar - könnte man einwenden - brutaler Unternehmerterror, aber

$7 \quad$ Inprekorr $1930, \mathrm{Nr} .25, \mathrm{~S} .586$

8 Manuilski. Bericht auf dem 11. EKKI-Plenum, Hamburg-Berlin 1931

8a Inprekorr 1930, Nr. 14, S. 327. Dieser Erlaß wurde auch durchgeführt. Vgl. dazu Inprekorr 1930, Nr. 10, S. 231 
noch kein Sozialfaschismus. Obwohl ich grundsätzlich auch diesem Einwand skeptisch gegenüberstehe, zeigt die Rede des späteren SPD-Vorsitzenden Otto Wels auf dem Magdeburger Parteitag von 1929, daß die SPD-Führung durchaus selbst zur sozialfaschistischen Diktatur über die Arbeiterklasse bereit war. Er sagte dort:

„,Es ist unsere Aufgabe, die Demokratie zu sichern, die Republik zu schützen. Gelänge es aber den Feinden der Republik, der Demokratie in Deutschland schweren Schaden zuzufügen, da kein anderer Ausweg bliebe als die Diktatur, dann sollen Stahlhelm, Nationalsozialisten und ihre kommunistischen Brüder von Moskau wissen: Die Sozialdemokratie und die Gewerkschaften als die Vertreter der großen Masse des deutschen Volkes, festgefügt in ihren Organisationen, in verantwortungsbewußtem Handeln und unzerbrechlicher Disziplin würden auch sie (also die Diktatur) zu handhaben wissen. Das Recht der Diktatur fiele ihnen zu und niemand anderem“" (9).

Damit keine Mißverständnisse aufkommen: die sozialfaschistischen Führer der SPD machten sich nur in begrenztem Umfang am Ende der Weimarer Republik selbst die Finger offen schmutzig; sie mußten ihre Funktion erfillen, einen großen Teil der Arbeiterklasse an den immer terroristischer werdenden Staat zu binden. Als die SPD einen Teil ihrer Stimmen verlor, als Teile der SPD die Politik ihrer Führer nicht mehr mitmachten, mußten andere Charaktermasken die Regierungsverantwortung für die Verelendungspolitik übernehmen.

Hatten diese Widerspriiche zur eigenen Basis jedoch zur Folge, daß die SPDund ADGB-Führer sich nicht mehr an der Abschaffung des bürgerlichen parlamentarischen Staates, nicht mehr an der Zwangsschlichtungspolitik und an dem Terror gegenüber der Arbeiterklasse beteiligten? Diese Frage ist mit einem eindeutigen Nein zu beantworten.

Seit 1930 herrschten bis zur ,Machtergreifung ${ }^{6}$ Hitlers nur noch Minderheitskabinette mithilfe von Notverordnungen, die die Weimarer Verfassung faktisch außer Kraft setzten. Rosenberg sagt richtig: nach 1930 hatte die Weimarer Republik aufgehört zu existieren. Und diese Notverordnungsregime hielten sich nur Dank der offenen oder versteckten Unterstützung der SPD-Führer. So erklärten die Häupter der SPD gegenüber dem Reichskanzler Brüning, daß ,sie keine Bedenken gegen diktatorische Vollmachten" hätten. Und die SPD-Führer waren es, die den Reichspräsidenten Hindenburg in seiner zweiten Wahl unterstützten als „Hüter der Verfassung", obwohl gerade er die Notverordnungen unterzeichnete, ja sogar soziale Wahlen per Notverordnungen verbot und schließlich Hitler zum Reichskanzler ernannte.

Sogar dann noch, als Teile der Bourgeoisie die SPD offen bekämpften, als sie sich mehr und mehr auf die faschistische Faust und ihre soziale Basis verließen, hielten die SPD-Führer ihren Herren die Stange: deutlich sichtbar in Preußen, ebenso deutlich sichtbar im Reichsrat, wo der Vertreter Preußens repräsentiert war und die Minderheitskabinette unterstützte. So erklärte Braun, es sei falsch, Brüning zu stürzen. Wem all dies zu wenig ist, um die Wegbereiterfunktion der SPD-Führer für den Faschismus zu akzeptieren, der sei ebenfalls an Braun, den führenden Sozialdemokraten seiner Zeit, verwiesen, der kurz nach seiner Absetzung als Ministerpräsi-

9 Zit. nach: Protokoll der Verhandlungen des 12. Parteitages der KPD, Berlin-Wedding, S. 74 
dent Preußens 1932 die NSDAP in eine Koalitionsregierung zu holen gedachte: man müsse den ,wundergläubigen Massen die Eisenbartkur des Nationalsozialismus" verordnen, damit sie erkennen, daß der Faschismus in seinen Regierungstaten schnell scheitern würde (10).

Schon am 3. Dezember 1931 wurde in einem Leitartikel des sozialdemokratischen „Vorwärts" erklärt, daß man die Nazis ,lieber heute als morgen an die Regierungsmacht, heranlassen" ", wenn diese die Spielregeln der Demokratie . . . beachten $^{6 ;} ;$, der ganze Schwindel wäre dann sehr schnell zu Ende."

Und seit November 1931 lehnte die SPD- und ADGB-Fühnung sämtliche Einheitsfrontangebote der KPD und RGO ab und unterstützte faktisch die Besetzung parlamentarischer oder staatlicher Verwaltungsstellen durch Faschisten, indem sie keine eigenen Kandidanten aufstellten, die von der KPD unterstiitzt worden wären (z. R. Reichstagspräsident). Sie lehnte nicht nur einen gemeinsamen bewaffneten Widerstand ab, sondern sogar den gemeinsamen Generalstreik am 21.1.1933.

Und nach dem 30. Januar 1933 zog eine Delegation der SPD durch andere Länder Westeuropas, um andere Parteien der Zweiten Internationale davon abzuhalten, über die faschistischen Terrormaßnahmen zu berichten, damit die Sozialdemokratie und die Gewerkschaften nicht gefährdet würden (11).

$J a$, die SPD-Führung schloß 1933 sogar ganze Ortsgruppen ihrer Jugendorganisation SAJ aus, weil sie mit dem ,illegalen" Kampf gegen den Faschismus begannen (11a).

Meiner Ansicht nach stellt Niels Kadritzke in der geraden Nachfolge Trotzkis in kruder Weise ,die" Demokratie ,dem“" Faschismus gegenüber, zieht einen tiefen Graben zwischen ,, der" „reformistischen ${ }^{66}$ Partei und der NSDAP. Dieser tiefe Graben existierte nicht mehr, da ,die" bürgerliche Demokratie schon nicht mehr bestand, als der Faschismus auf legale Weise die Macht übernahm. In der tiefen ökonomischen und politischen Krise des Kapitalismus und seines Staates verteidigten sämtliche bürgerlichen Parteien einschließlich der SPD mit terroristischen Mitteln den bürgerlichen Staat. Ich unterschätze dabei keineswegs den qualitativen Sprung zwischen Hitler- und Präsidialregierungen.

Die Unrichtigkeit der Theorien von der prinzipiellen Unvereinbarkeit von Faschismus und Sozialfaschismus zeigt auch das Beispiel des Pilsudski-Polen, in dem die Gewerkschaften formal unangetastet blieben und zur ,Agentur des Faschismus ${ }^{66}$ in der Arbeiterklasse wurden (Manuilski).

Der Terror, mit dem die SPD-Führung die Abwälzung der Krise auf die Werktätigen zusammen mit anderen bürgerlichen Parteien politisch durchsetzte, die nationalistische, die Volksgemeinschaftsideologie, die Leier vom gemeinsamen Gürtel, den es enger zu schnallen gilt, sogar der rigorose Abbau demokratischer Rechte unter der Regierung Müller und mithilfe der SPD- und ADGB-Führung während der Präsidialregime und die Wegbereiterfunktion der SPD- und ADGB-Führung für den

10 Jürgen Bay, der andere ,20. Juli“", in: Die Zeit, Nr. 29 yom 21.7. 1972, S. 40

11 Chronik zur Geschichte der deutschen Arbeiterbewegung, Bd. 2, Berlin/DDR 1966, S. 316

11a Braunthal, Julius, Geschichte der Internationale, Bd. 2, Hannover 1963, S. 404 
Faschismus - das alles für sich genommen, ist für mich noch nicht ausschlaggebend, um die Richtigkeit der Einschätzungen über den Sozialfaschismus zu beweisen. Es kommt ein Moment hinzu, das im Programm der heutigen KPD folgendermaßen bestimmt wird; ich zitiere sinngemäß:

Die sozialdemokratischen Führungsgruppen können bei einer tiefgreifenden ökonomischen und politischen Krise des Kapitalismus und seines Staates zum Sozialfaschismus verkommen: entweder in der Form der von ihnen getragenen terroristischen Diktatur über die Arbeiterklasse oder in der Form der Arbeitsteilung mit einem regierenden Faschismus oder als Wegbereiter des Faschismus. Diese möglichen Entwicklungen werden von dem Stand der Klassenkräfte und von der Monopolbourgeoisie abhängen und davon, ob es der SPD-Führung gelingen wird, den reaktionären Gewerkschaftsapparat in das terroristische Unterdrückungssystem einzugliedern.

Wie verhielt sich also der Gewerkschaftsapparat vor dem Sieg des Faschismus und zu Beginn seiner Regierungszeit? Bei der Beantwortung dieser Frage komme ich zu

\section{Kadritzkes 2. These}

Die SPD- und ADGB-Führung war niemals zu einer Arbeitsteilung mit dem regierenden Faschismus bereit.

Demgegenüber meine These: Die ADGB-Führer (teilweise auch die SPD-Führung) hofften auf ein Arrangement und eine Arbeitsteilung mit den Faschisten. Nachdem die ADGB-Führer, ideologisch und teilweise in Personalunion mit der SPD-Fühnung verwachsen, von 1929 bis 1933 in Zwangsschlichtungen den Lohnraub sanktionierten, die Großen Streiks abwürgten und für illegal erklärten, Streikbruch organisierten, Kommunisten und andere Streikleiter denunzierten, sich gezielt an der Rausschmißßpolitik gegen Kommunisten beteiligten, ganze Ortsverbände aus dem ADGB ausschlossen, Gewerkschaftswahlen annulierten und andere demokratische Rechte der Gewerkschaftsmitglieder außer Kraft setzten, waren die ADGB-Führer mindestens seit dem 9. September 1973 bereit, den faschistischen Vorstellungen von einer verstaatlichten ständischen Gewerkschaft Vorschub zu leisten.

Am 9. September 1932 nämlich, also nicht erst unter Reichskanzler Schleicher, sondern bereits unter Papen, trafen sich zu Verhandlungen: ein Vertreter Papens, Schleicher (Reichswehrminister), Vertreter der faschistischen Gewerkschaften und Vertreter des ADGB. Um die Ungeheuerlichkeiten der ADGB-Politik dieser Jahre zu demonstrieren, muß ich eine längere Passage aus dem Verhandlungsprotokoll zitieren:

„....der Wirtschaftstheoretiker der NSDAP, Herr Wagner,... entwickelt seine bekannten Ideen von den ständischen Aufgaben der Gewerkschaften, die in ihrer heute rein klassenkämpferischen (!) Gestalt vom Wesen der wahren Gewerkschaft, d. h. Zusammenschluß einer Arbeitsund Arbeitergemeinschaft, sich allzu weit entfernt hätten ... Die praktische Konsequenz dieses Grundsatzes sei der Einbau der Gewerkschaften in den staatlichen Apparat. Zuerst Zusammenfassung aller bestehenden Arbeitnehmerorganisationen nach Fachgebieten, sodann eine Art Zwangsmitgliedschaft und dann Übernahme des gesamten dazu notwendigen Apparates durch 
den Staat....

Sodann sprach äußerst vorsichtig und zurückhaltend Herr Eggert (ADGB), der ohne weiteres erkennen ließ, daß er grundsätzlich mit dem Ziele der Verstaatlichung einverstanden sei. Er machte dann aber auf die außerordentliche Schwierigkeit der Zusammenfassung so heterogener Elemente aufmerksam. (Zwischenruf des Herrn Wagner: Es handelt sich ja dann um eine Behörde, was allseitig (!) mit Heiterkeit quittiert wurde.) Eggert glaubte, daß es praktischer sei, zuerst mit einer Machtanhäufung der Gewerkschaften zu beginnen, bevor man an die Verstaatlichung herangehe. Die Gewerkschaften, für die er spreche, könnten nicht so ohne weiteres auf die wertvollen, jetzt noch revolutionären Kräfte verzichten, die bei einer offenen (!) Verstaatlichung unter ständischen Gesichtspunkten sich einem dahin zielenden Zwang mit allen Mitteln widersetzen würden. Andererseits sei bei der Hereinnahme dieser Kräfte mit deren Überhandnehmen und einem Durchkreuzen der vorgetragenen Absichten in bolschewistischem Sinne zu rechnen. Man muß daher allen Organisationen Zeit lassen, diesen Gedankengängen assimiliert zu werden. Durch die Ideologie der Gewerkschaften (!) sei die Assimilation schon weit, aber noch nicht weit genug fortgeschritten...

Zum Schluß dankte Herr von Schleicher nochmals und betonte die erfreuliche sachliche Ủbereinstimmung ..." (12)

Diese Protokolle zu Verhandlungen, die noch nicht unter faschistischem Terror zustandekamen, machen verschiedenes deutlich:

- Die ADGB-Führer wollten ebenfalls eine Verstaatlichung und Umwandlung der Gewerkschaften in ständisch-faschistischem Sinne;

- $\quad$ es ging nur noch um das Wie dieser Umwandlung; die ADGB-Führer hatten vor allem taktische Bedenken und wollten sich Zeit lassen, ihre Mitglieder mit den Vorstellungen faschistischer Gewerkschaften vertraut zu machen;

- die ADGB-Führer schätzten sich vollkommen richtig ein, wenn sie ihre ideologische Vorarbeit für faschistische Gewerkschaften (,Assimilation") betonten. In dieser Frage gab es allerdings Widersprüche zur SPD-Führung. Trotzdem zeigt die gesamte Politik der ADGB-Führer nach dem 30. Januar 1933, daß sie zu einer faschistischen Gewerkschaft und zu einer Arbeitsteilung mit dem regierenden Faschismus bereit waren.

(Auf das meiner Ansicht nach ebenfalls sozialfaschistische Arbeitsbeschaffungsprogramm und die Vorstellungen der SPD- und ADGB-Spitze zum Arbeitsdienst kann ich hier nicht eingehen.)

Nach verschiedenen Erklärungen, in denen sie betonten, die sozialen Aufgaben der Gewerkschaften müssen erfuillt werden, ,gleichviel welcher Art das Staatsregime is $t^{66}$, traten die ADGB-Führer am 5. April 1933 ,,in Verhandlungen mit der NSDAP zur Vereinheitlichung des deutschen Gewerkschaftslebens" ein. In diesen Verhandlungen ging es ebenfalls nur noch um das Wie: die NSDAP-Vertreter traten selbstbewußter auf und stellten klar die Forderung nach der Führung. Einer der Führer des ADGB, Franz Grosse, schrieb am 15. April in der ,Gewerkschaftszeitung ${ }^{66}$ eine Aufforderung zur Arbeitsteilung an die NSDAP: die neuen Machthaber müßten erkennen, daß sie in ihrem eigenen Interesse das faschistische Beispiel der Unterdrückung der Gewerkschaften (Italiens) nicht befolgen dürften: „Nur unter Berücksichtigung der auf eigener völkischer Grundlage gewachsenen Kräfte " kann "die nationale Regierung das fremde Vorbild nicht nur erreichen, sondern .. auch

12 Zit. nach Hannes Heer. Burgfrieden oder Klassenkampf, Neuwied 1972, S. 159 ff. 
ubertreffen ${ }^{64}$ (13).

Das war eine offene faschistische Sprache. Und all dies zeigt deutlich, daß die ADGB-Führer zu Sozialfaschisten geworden waren, eben nicht erst unter dem terro. ristischen Druck der regierenden Faschisten, sondern bereits seit 1932.

Einige Führer des ADGB hatten auch die parteipolitische Konsequenz gezogen: so trat der Leiter der zentralen ADGB-Schule in Bernau, Herman Seelbach, in die NSDAP ein. Er selbst schreibt über seinen Weg zur NSDAP, daß es bei der Umwandlung der Gewerkschaften seitens der ADGB-Führer keine nennenswerten Widerstände gegeben habe, daß viele Führer des $\mathrm{ADGB}$ auf der richtigen (der faschistischen).Seite gestanden hätten (14). Uber die Umwandlung seien sich alle einig gewesen, nur das Wie war auch innerhalb der NSDAP strittig. Hatte der, ,Führer ${ }^{66}$ noch am 5. April die Zusicherung gegeben, die Gewerkschaften nicht zu enteignen, so wurde am 2. Mai 1933, einen Tag nach dem auch von der ADGB-Führung unterstiitzten ,Tag der nationalen Arbeit ${ }^{66}$, das Eigentum der Gewerkschaften verstaatlicht und die Umwandlung der Gewerkschaft in die DAF erfolgte durch die Staatsgewalt: die Faschisten hatten es nicht mehr nötig, auf ihre Führungsrolle gegenüber den total verrotteten ADGB-Führem zu verzichten. Mit Widerstand brauchten die Faschisten nicht zu rechnen, da sich im Kern, nämlich in der Frage der Umwandlung, alle einig waren.

An dieser Stelle verkommen zwei ,Argumente ${ }^{66}$ Kadritzkes vollends zur Absurdität:

Weil die Gewerkschaften verstaatlicht wurden durch faschistische Regierungsgewalt könnten sich die ADGB-Führer gar nicht zu Sozialfaschisten, zu Wegbereitern des Faschismus, entwickelt haben. Das ganze eben angeführte Material beweist das Gegenteil. Es ging nur noch um die Führungsrolle. Der Mohr hatte seine Schuldigkeit getan.

Als zweites ,Argument ${ }^{66}$ fihrt Kadritzke ins Feld: ich verwechsle ständig die objektive und die subjektive Ebene: auch wenn die ADGB-Führer subjektiv zu einem Arrangement mit den Faschisten bereit waren, konnten sie doch objektiv die Vestaatlichung in die faschistische Ständegewerkschaft nicht durchführen, das sei den Faschisten vorbehalten geblieben. Dieses, Argument ${ }^{6 /}$ zeigt eine erschreckend mechanistische Bestimmung des Begriffs ,objektiv ${ }^{66}$ : es geht nicht nur bei der Bestimmung des Begriffs , ${ }_{\text {objektiv }}^{66}$ um die Haltung der Kapitalisten, sondern die ,objektive Funktion ${ }^{66}$ der ADGB-Führer war es gerade, den Faschisten bei der Eingliederung der Gewerkschaften in den faschistischen Staat bzw。 in die DAF zu helfen. Diese ,objektive ${ }^{66}$ Funktion zu erfüllen, waren die ADGB-Führer auch subjektiv bereit.

Wer über diese Ungeheuerlichkeiten der ADGB-Führer schweigt oder sie als, opportunistisch", als Ausdruck der Angst vor dem Faschismus verharmlost, wer permanent über diese Politik der ADGB-Führer schon vor 1933 wider besseres Wissen kein Wort verliert, der leistet seinen ,linken" Beitrag zu den sozialdemokratischen Ge-

14 Hermann Seelbach. Das Ende der Gewerkschaften, Berin 1934 
schichtsfälschungen, wie wir sie gerade angesichts der 40jährigen Wiederkehr der faschistischen ,Machtergreifung" aufgetischt bekommen.

\section{Kadritzkes 3. These}

Die führenden Sozialdemokraten hätten keine Staatstheorie vertreten, nach der sie den demokratischen Staat und die „Planungs"elemente im Kapitalismus als Übergangsform zum Sozialismus begriffen.

Dagegen meine Feststellung: die SPD-Führer gingen am Ende der Weimarer Republik von einem Staat aus, der aus ökonomischer Notwendigkeit das , sozialistische Prinzip der Planung" innerhalb des Kapitalismus entwickelt habe. Diese Staatstheorie - Sozialismus innerhalb des Kapitalismus - mußte in der tiefen Krise der faschistischen Staatstheorie nahekommen.

Belege für diese These: Zunächst Hilferdings Rede auf dem Kieler Parteitag 1927, auf dem er sagte:

„Organisierter Kapitalismus bedeutet also in Wirklichkeit den prinzipiellen Ersatz des kapitalistischen Prinzips der freien Konkurrenz durch das sozialistische Prinzip planmäßiger Produktion. Diese planmäßige, mit Bewußtsein geleitete Wirtschaft unterliegt in viel höherem Maße der Möglichkeit bewußter Einwirkung der Gesellschaft, das heißt nichts anderes, als der Einwirkung durch die einzige bewußte und mit Zwangsgewalt ausgestaftete Organisation der Gesellschaft, der Einwirkung durch den Staat" (15).

Kadritzke behauptet nun, diese Vorstellungen seien in der Krise aufgegeben worden. Das ist falsch, wie sich aus zahlreichen Dokumenten ergibt. Braunthal, ein führender Kopf der Sozialdemokratie, sprach auch 1931 vom ,schmerzlichen Übergangszustand $^{\text {"6 }}$ vom Staatskapitalismus zum Sozialismus, und Hilferding selbst verteidigte die staatlichen Aufkäufe der bankrotten Gelsenkirchen AG durch den Staat 1933 und sagte, damit sei ein ,ganzes Stück Marxismus vollzogen" (16). Und Naphtali, ein führender Gewerkschafter, erklärte die staatliche Subventionspolitik als „,Hilfestellungen des Staates für den Umbau des Staates in sozialistischem Sinne ${ }^{66}$ (17). Und für Braunthal sind es gerade die Krisenerscheinungen und die ständigen Eingriffe des Staates in die Wirtschaft, die die Voraussetzungen für den staatlichen Umbau zum Sozialismus schaffen.

Die Kette dieser Argumente ließe sich beliebig fortsetzen. Besonders deutlich wird diese Argumentation nach der Veröffentlichung des Roosevelt-Programms, das in der gesamten Zweiten Internationale als Übergang zum Sozialismus gefeiert wurde. Und Otto Bauer, der führende Kopf der Zweiten Internationale, schrieb noch 1930, „daß jedes Stück Sozialismus, das wir heute schon erobern, den Bereich der

15 Protokolle des Kieler Parteitags, Raubdruck, S. 168

16 Braunthal, in: „Leipziger Volkszeitung" vom 24. 12.1931. Hilferding zit. nach A. Fogarasi: Der Bankrott der Theorien des Sozialfaschismus am Ende der kapitalistischen Stabilisierung, Moskau-Leningrad 1934, S. 37 Ebd., S. 38 
kapitalistischen Anarchie . . einschränkt" (18). Staatliche Eingriffe als Elemente des Sozialismus - das sind die Kernthesen der gesamten sozialdemokratischen Staatstheorien auf der Grundlage des ,organisierten Kapitalismus oder des Staatskapitalismus".

Diese Kernthesen sind besonders gefährlich in einer Situation der akuten Bedrohung durch den Faschismus, der ebenfalls einen Sozialismus propagierte, ohne die bolschewistische Revolution und ohne die ,Eigentumsfrage“ zur Hauptfrage zu machen. So schrieb der Sozialdemokrat Vandervelde: „Das große Problem des Sozialismus ist im Moment nicht so sehr das Problem des Eigentums, als vielmehr der Freiheit" (19).

Mir ist nicht unbekannt, daß das Scheitern der ,Reformpolitik ${ }^{66}$ seit 1929 ein hilfloses Wuchern ,neuer" Staatstheorien innerhalb der Sozialdemokratie hervorrief. Ich kann wegen der Kürze des mir zur Verfügung gestellten Raumes nur die „linken“ Sozialisten auf dem Kongreß der Sozialistischen Partei Frankreichs (Juli 1933) zitieren, die den ,Rechten ${ }^{6}$ zuriefen: „Thr seid Reformisten ohne Reformen!" Worauf sie die passende Antwort erhielten: „Und ihr seid Revolutionäre ohne Revolution!"“(20)

Ebenfalls wegen der Kürze des Raumes kann ich nur knapp eingehen auf

\section{Kadritzkes 4. These}

Die Wirtschaftsdemokratie der SPD und ADGB-Führung war reine reformistische Illusionsmacherei - nicht mehr.

Dazu meine Stellungnahme: in der Tat - die Wirtschasftsdemokratie war Illusionsmacherei vor der Krise. (Hier habe ich auch eine kritische Haltung gegenüber der Darstellung der KPD und Komintern.) Worum es aber geht, ist folgendes: Die wirtschaftsdemokratischen Vorstellungen basierten auf eben der Theorie vom organisierten Kapitalismus, auf eben der Staatstheorie, die staatliche Umverteilung, verbunden mit langfristiger Planung, als sozialistisches Prinzip feierte. In der Krise mußte diese Theorie umschlagen in die Praxis der staatlichen Umverteilung zu Lasten der Arbeiterklasse und zu Gunsten der Subvention des Kapitals.

Genau dies tat die SPD- und ADGB-Führung. Sie sanktionierten den Lohnraub in Zwangsschlichtungen und faktischer Arbeitsgemeinschaftspolitik, auch wenn - worauf Kadritzke triumphierend hinweist - die sog. Arbeitsgemeinschaften aus Kapital, Staat und Gewerkschaftsführung nach bisherigen Quellen wohl nur einmal unter diesem Namen im Juni 1930 zusammentraten. Aber es geht um die Praxis und Kadritzke fällt herein auf die SPD-Ideologie, wenn er die gesamte staatliche Zwangsschlichtung unter Beteiligung der SPD- und ADGB-Führer, ihre gesamte Lohnraubpolitik nur deshalb nicht als Arbeitsgemeinschaftspolitik bezeichnet, weil die Gewerkschaftsführer sie nicht so nannten.

18 Ebd., S. 51

19 Zit. nach ,Unter dem Banner des Marxismus“, 1934, Heft 1, S. 79

20 Ebd., S. 60 
In dem Arbeitsgemeinschaftstreffen im Juni 1930 wurde festgelegt, daß , in der Volkswirtschaft alle Störungsmomente möglichst aus(ge)schaltet, insbesondere die Gefahr von Arbeitskämpfen auf ein Minimum reduziert ${ }^{66}$ werden sollien (21).

Auch wenn die Gewerkschaftsführer nicht mehr von Arbeitsgemeinschaften sprachen, so verhielten sie sich doch danach. Oder wie erklärt sich Kadritzke, daß sich die ADGB-Führer an allen Zwangsschlichtungen beteiligten, die die Löhne weit unter das Existenzminimum drückten? Und daß die ADGB- und SPD-Führer selbst in den staatlichen Schilchtungen saßen. Indem Kadritzke die ,reformistische" Ideologie in den Vordergrund und die Praxis der Bonzen in den Hintergrund rückt, verhält er sich mindestens als schlechter Intellektueller. Daß diese faktische Arbeitsgemeinschaftspolitik eben die gleiche Funktion wie die faschistischen Arbeitsgemeinschaften in der Krise erhalten und teilweise auch ähnlich begründet wurden, gerät Kadritzke so vollends aus dem Blickfeld.

Zum Schluß kann ich nur bedauernd feststellen, daß angesichts der mir auferlegten Kürze nur ein Teil der Probleme angegangen werden konnte. Ich bin aber gerne bereit, zu den hier nicht behandelten Punkten in weiteren Aufsätzen Stellung $\mathrm{zu}$ beziehen, so z. B.

- zu der KPD-Politik 1928 bis 1933, insbesondere zu der Frage, warum die Bolschewisierung und die „Organisationsfrage", die Kadritzke so unterschätzt, für uns eine so zentrale Bedeutung besitzt gerade für die Gewinnung der Arbeiterklasse und anderer unterdrückter Schichten des Volkes; (unerhört finde ich die Gleichsetzung der „Schuld“ von SPD und KPD für den Sieg des Faschismus bei Kadritzke);

- zu dem Vorwurf, die „Theorie“" vom Sozialfaschismus habe zur „Faschismus-Blindheit" geführt;

zu der Frage, warum wir die jetzige SPD-Politik nicht als sozialfaschistisch bezeichnen;

- $\quad$ zu der Bedeutung des „Begriffs“ Sozialfaschismus als Kampfbegriff. Ist es immer nơwendig, einen analytisch für richtig gehaltenen Begriff in der Propaganda zu benutzen, wenn er den sozialdemokratischen Mitgliedern noch nicht klar ist, weil er ein Begriff der Marxisten-Leninisten ist? (Inzwischen würde ich diese Frage bejahen.)

- Insbesondere würde ich gerne klarmachen, daß fast alle Kritiker des, ,Sozialfaschismus-Begriffs" in heutigen strategischen Diskussionen ausschließlich vom klassischen Faschismus als der einzig möglichen faschistischen Entwicklung ausgehen. Gerade französische Kommunisten (z. B. Geismar) haben versucht, am Beispiel der USA oder Argentiniens den Zusammenhang von Staat und faschistischen Maßnahmen heute neu zu bestimmen.

Obwohl all diese Probleme hier nicht behandelt werden konnten, hoffe ich trotzdem, daß dem Leser schärfer der Kern der Auseinandersetzung um den Sozialfaschismus vor Augen getreten ist und daß die Einschätzungen der Kommunisten nicht leere oder dogmatísche Behauptungen sind, sondern Realität der Politik der SPD- und ADGB-Führung treffen: daß diese aktiv und terroristisch Wegbereiter des

21 Deutsche Allgemeine Zeitung vom 4, 6.1930 
Faschismus waren, daß sie rechtliche und ideologische Vorbedingungen für den Sieg des Faschismus schufen, đaß sie Elemente der faschistischen Ideologie aufnahmen und mit terroristischen Mitteln die Verelendung der Arbeiterklasse durchsetzten.

Kadritzke sollte Farbe bekennen und Antwort auf die Frage geben, warum er permanent den SPD-Ideologen oder Brüning mehr Glauben schenkt als den Kommunisten, warum er ständig die Theorie und Praxis der SPD- und ADGB-Führer verharmlost oder verschweigt. Wem nützt diese Haltung? Es geht heute darum, die Verbrechen der SPD- und ADGB-Führung wieder ins Gedächtnis zurückzurufen!

\section{Materialien und Untersuchungen zu Kapital- bewegung. Staatsapparat und Klassenanalyse in den Industriezentren der $B R D$ und der $E G$}

\section{Harry W. Jablonowski, Johanna Offermanns \\ Teilkrise ohne Ende}

Eine Untersuchung über den industriellen "Strukturwandel" im Ruthrgebiet Welche Kapitalbewegungen stecken hinter der sogenannten "Strukturkrise" im größten industriellen Ballungszentrum Europas? Mit welchen Strategien antworten Kapital und Staat auf die verschlechterten Verwertungsbedingungen in einzelnen Wirtschaftssektoren und Regionen (Zentralisation, Rationalisierungen, Entlassungen, Kapitalexport usw.)? Welche Konsequenzen hat diese Teilkrise für die Arbeiterklasse?

Unter diesen Fragestellungen werden zahlreiche Informationen aus Prognosen, Statistiken und Berichten wirtschaftswissenschaftlicher Forschungsinstitute usw. aufgearbeitet und gleichzeitig einige wichtige Untersuchungsmethoden der bürgerlichen Regionalanalyse kritisiert.

Die Arbeit kann also

1. über Methodenkritik hinaus Argumente gegen die Theorie und Propaganda von der Krisenelastizität des kapitalistischen Systems liefern;

2. methodische Anregungen für die Erstellung politökonomischer Analysen geben;

3. zur materialistischen Einschätzung der zahlreichen Teilkrisen in Industriezentren der EG, Japans und der USA beitragen, deren Charakter der bürgerliche Begriff "Strukturkrise" verdeckt.

Das Buch erscheint im Verlag des Politladen Erlangen im Herbst 1974. Ca. 340 Seiten, ca. 13,00 DM. 